\title{
GLOBAL 3D-GRIDS BASED ON GREAT CIRCLE ARC QTM SPHERE OCTREE AND ITS APPLICATION
}

\author{
Jinxin Wang ${ }^{\text {a, }}$,Yaohui Li $^{\text {a }}$, Yasheng Zheng ${ }^{\text {a }}$, Junnan Liu ${ }^{\text {a }}$ \\ ${ }^{a}$ School of Water Conservancy \& Environment of Zhengzhou University, Zhengzhou, 450001, China - \\ jxwang@zzu.edu.cn, (799723343, 1424236433, 6929423)@qq.com
}

KEY WORDS: Discrete Global Grids (DGGs), Earth System Spatial Grid ( ESSG), Quaternary Triangula Mesh (QTM), Global GIS, Spatial Data Model, Digital Earth

\begin{abstract}
:
With the development of computers, network communications, scientific computing, mapping remote sensing and geographic information technologies, Discrete Global Grids (DGGs) and Earth System Spatial Grid (ESSG) have become the integrated spatial data model facing the large-scale and global-scale problems and the complex geo-computation. This paper discusses the property and character of the global spatial data at first. Then it introduces the grid division system based on large arc QTM octree and compares this scheme with degradation octree scheme. At last, it introduces the application of the scheme in land surface, underground and aerial geographic entity modeling. The study suggests that: the grid division system based on large arc QTM octree has the potential to integrate the whole spatial data of different layers of the geospatial. And it will have a broad application prospect in complex large-scale geographic computing.
\end{abstract}

\section{INTRODUCTION}

Physically, the earth is an ordinary celestial in the universe. Geospatial is a three-dimensional, multi-circle manifold spatial domain within the gravity field, including the Earth's inner core, outer core, lower mantle, upper mantle, crust, atmosphere, ionosphere and magnetosphere, etc., and all the phenomena and processes of the objective world have taken place in this space. However, for the past thousands of years, Euclidean geometry, the simplest abstract of the space, has become a commonly used tool for understanding the objective world because of the limitation of the human awareness and technical levels. People formed two ingrained habits of mind in understanding the geospatial from the Euclidean geometry point of view: The first habit is to use two-dimensional Euclidean coordinates (latitude and longitude) to locate the position of geographical entities and phenomena. From the mathematical perspective, it is imprecise to use latitude and longitude to define the position of the sphere, because the longitude of bipolar points does not exist. we cannot get the latitude and longitude coordinates of the pole; the second habit is projection. As a symbol of human civilization, maps based on the projection have brought convenience to people's daily application. But in the information age, the projection brought three problems to geospatial information: data crack, inaccurate spatial analysis, difficulties in data integration and sharing(Lixin,W., et al, 2009). At present, the GIS spatial data model is ultimately the European projection model.

Logically, the Earth system is a nonlinear complex giant system formed by the spheres of the Earth and interaction among them, and the "butterfly effect" is a typical example. On the one hand, with the continuous development of social economy, global issues have become increasingly prominent; On the other hand, people gradually realize that from the overall, integrated, multi-dimensional perspective to reveal the large-scale and global-scale geographic rule is a major issue humanity now facing. Essentially, in the background of IT, global scale Geo-computation is a super complex problem with data-intensive, knowledge-intensive and computing-intensive. The traditional projection-based GIS spatial data model can not meet this complex application. Thus it is imperative to develop the integrated spatial data model framework meeting the needs of global scale, mass data, and the large computing capacity. Technically, digital earth is a technical architecture which integrates the spatial data with multi-resolution, multitemporal, multi-form and multi-sources, and also a platform for people to simulate, deduce and reappear the objective geography process. But now, the digital earth is still confined to the modeling of the surface of the earth. With the development of remote sensing, telemetry and other modern mapping technology, people's ability to get massive spatial data space has improved, entered the "big geographic data" era. Meanwhile, with the greatly enhance of the computer storage capacity and computing power and the developing of sensor networks, the internet of things, cluster computing, pervasive computing, grid computing, cloud computing and other new techologies, the global-scale super-complex Geo-computation has become possible. Human society entered the era of Digital Earth and Smart Earth. however, GIS spatial data model, the core of complex Geo-computation, developed relatively slow. So it is very important and necessary to create new spatial data model that orient intelligence computation.

The so-called integrated and new data model has two basic requirements: Firstly, it can restore manifold space characteristics of the earth's gravitational field. Secondly, it should have the ability to integrate the spatial data of the whole geography space range from magnetic layer to geocentric. One possible approach is dividing the gravitational field into

\footnotetext{
* Corresponding author.
} 
many discrete manifolds (or Euclidean) "sub-block", then using these sub-blocks as "elements" to approximate the manifold space and its entities. By doing this, it can be used as the foundation to organize and manage the large-scale, global, massive geospatial data. Thus, the global discrete grid (Discrete Global Grids, DGGs) has been a research direction in geographic information science. Especially in recent years, the field has made large research achievements. Just as Goodchild, M F. (2000) said, with its excellent features like unique hierarchy, regularity, clarity in spatial resolution and consistency in global expression and so on, DGGs is an indispensable technical tools and methods to digital earth by providing geospatial reference, spatial indexes, and discrete spatial data sets for it.

Strictly, the global discrete gird includes the traditional surface grid and extend solid grid. There are nearly 20 kinds of specific subdivision schemes that belong to the spherical surface grid like the classic latitude and longitude grid, Voronoi grid, grid-based regular polyhedron and so on. Among all the schemes, the gird based on regular octahedron has the characters of clear positioning, relatively simple structure and moderate deformation, and it is the most researched and applied discrete gird data model( Zhao, X. S., et al, 2002). Types of spherical solid grid are relatively few, only eight kinds, including latitude and longitude grid, the radially extending spherical triangular mesh grid [Baumgardner, J R., et al, 1985], the Cubed-sphere grid [Tsuboi, S., et al, 2008], the "Yin Yang grid" [Kageyama, A., et al, 2004], the based on triangulation and radial spherical discontinuity divided grid [Ballard, S., et al, 2009], the adaptive mesh refinement (Adaptive Mesh Refinement, AMR) [Stadler, G., et al, 2010], the degradated octree grid[Lixin, W., et al, 2009], and the great arc QTM octree grid [Jinxin, W., et al, 2012;2013] . From the following discussion shows that the Grids which fit for GIS spatial data model, or the universal Earth system (Lixin, W., et al(2012) called it as Earth System Spatial Grid, ESSG) only are latter degenerated octree and octree .

This paper firstly discusses the characteristics that the discrete grid GIS spatial data model ( or ESSG) should have based on the mathematical cognitive on manifold space; Then, focus on octree subdivision method, characteristics, encoding and decoding scheme and its comparison with degenerated octree grid; Finally this paper introduces the several applications of octree grid.

\section{CHARACTERISTICS OF ESSG AS SPATIAL DATA MODEL}

\subsection{Characteristics of Euclidean plane and body subdivision}

Dimension of Euclidean space is flat and integral. A twodimensional plane is a abstract plane which is otropic, no thickness, and can be infinitely extended. It can be uniformly split by an ideal regular polygon (triangle, square, hexagonal, etc.). So you can use a uniform, homogeneous twodimensional Cartesian coordinate system to metrics plane grid. Any point on the plane can be mapped to a two-dimensional array. The shortest distance between two points is a straight line connecting two points . Commonly used raster GIS model is a typical discrete subdivision based on square cells. Euclidean three-dimensional space can be imagined as a cube of side length infinitely long, and you can use a unified, homogeneous three-dimensional Cartesian coordinate system to metrics. This space can be split as uniform cube grid system. Body split unit and surface split unit each has a consistent spatial relationship. In short, Euclidean space is a linear space.

\subsection{Characteristics of sphere surface and solid subdivision}

Sphere's surface and solid belong to non-Euclidean manifold space. They comply with Riemannian geometry, rather than Euclidean geometry. Nonlinear manifold sphere having different properties from planar. For example, spherical three angles of a triangle were greater than PI; Spherical only covered by approximately equal polygon, and cannot guarantee the distance between the side of any two adjacent grid points are equal .There aren't any base vectors which can represent all spherical grid point coordinates (Xuesheng, Z., et al, 2007). Similarly, the sphere body did not like the cube which can be splited into equal-area rule consistent grids, but it can be splited into the grid cells which have similar geometric characteristics at the same horizontal layer and large deformation at the radial (Globe direction) row; meanwhile, it also can not ensure the distance between any adjacent grid points are equal, and there aren't any base vectors can represent coordinates of all sphere grid .

Global discrete grid model is essentially a raster model; its uniformity of cells' size is critical. Non-uniform grid granularity effect the accuracy of spatial statistics, measurement and simulation, and finally it can lead to uneven data sampling, data redundancy and increasing amount of computation (Kimerling, A J., et al., 1999). Therefore, in order to achieve a relatively uniform of spherical grid system, people tried many different solutions. But these solutions, without exception, are to increase the complexity of cells deformation and their connection in exchange for their main geometric indicators are basically identical(Yongsheng, Z., et al., 2007) . These problems are essentially due to manifold characteristics of the ball. This method is to increase the storage space time and efficiency.

Spherical and plane aren't homeomorphic spaces, but the cube and sphere are. This mathematical properties of the subdivision meaning two things: First, there are also a simple shape of sphere solid grid cells which sides are all planar (as long as it's spherical edges are the great circle); second, Spherical subdivision exists a uniform benchmark metrics in the radial direction(Jinxin, W., et al., 2012). For example, radial uniform recursive subdivision can be achieved even equidistant subdivision. The properties is significant on the simplified or compressed 3D grids coding, and also make radially split has great flexibility, the Spherical and radial recursive split can be done separately, thus, you may choose the size of radial subdivision according to actual needs. Undoubtedly, it has a higher efficiency to performance the Earth's circle structure. In this paper, the great circle arc QTM octree subdivision will make full use of the mathematical properties of the sphere, so the scheme has many unique advantages.

\subsection{Characteristics of ESSG - earth system-oriented spatial data model}

As mentioned earlier, geospatial is a multi-zone manifold space of which the center is the centroid of the earth. The tightly coupled material and energy cycle among different spheres forms the complex giant nonlinear earth systems. As the organic characterization of geospatial structure and a 
spatial data model of geographic information science, ESSG should not only reflect the integrality and systematicness of geospatial and Earth system at first, but also have the carrying ability and inheritance. Thus, the geocentric, the equator, the prime meridian, circle structure and recursive subdivision are the important features of the ESSG model. From the earliest spherical subdivision standard of Goodchild(1994), to the proposed standard of spherical subdivision (Jinxin, W., et al, 2012), to Lixin,W., et al (2012) proposed basic requirements of ESSG, all of them reflect the same strain of the important characteristics of spatial data model. According to those standards, we can find that among the eight sphere schemes listed in front, the first six models are all single use grid system designed for particular field or application except for the latitude-longitude grid. They are unsuitable as the geographic information science (or geographic information systems) spatial data model because they are more serious violation of these standards subject (including latitude and longitude grid). Only the latter two models are in line with these criteria, which can be used as geographic spatial data model for complex Geo-compute oriented to the Earth system.

\section{GREAT CIRCLE ARC QTM OCTREE SUBDIVISION SCHEME}

\subsection{Subdivision method and its characteristics}

Our scheme can be divided into two relatively independent steps. The first step is the spherical subdivision. With $0^{\circ} \sim$ $180^{\circ}$ first meridian, east and west longitude $90^{\circ}$ meridian and the equator, the sphere is divided into eight equal area spherical triangles. Then, for each of the three sides of the triangle, midpoints were taken, and connected in order with a large arc to form four sublayer spherical triangles; and then based on this method (taking the midpoint of the arc sides, large circular connection), so after this all levels of recursive subdivision of spherical triangles, until it meets the application requirements (the number of levels of recursion $n$ ). The second step is radial split. From each node of the grid line to the center of the sphere (ball radius $\mathrm{R}$ ), depending on the application and select the appropriate radial distance, such as the radius of the aliquot to $\mathrm{R} / 2^{\mathrm{n}}$, at each hierarchical place with a spherical cut open (in center of the sphere as the origin to $\mathrm{k} *$ $\mathrm{R} / 2^{\mathrm{n}}$ drawing the ball radius, $\left.\mathrm{k}=1,2, \ldots \mathrm{n}-1\right)$. In this way, the ball can be divided into a spherical triangular units neatly and consistently (top or bottom wall is a spherical, planar side surface), which are the spherical triangular pyramids in center of the sphere(Left of Fig.1).

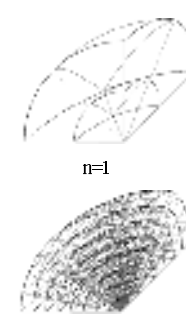

$n=3$

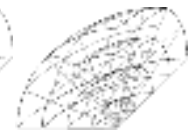

$\mathrm{n}=2$

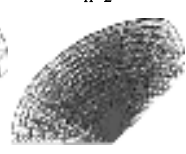

$\mathrm{n}=4$

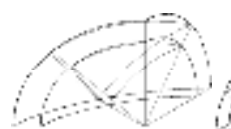

$\mathrm{n}=1$

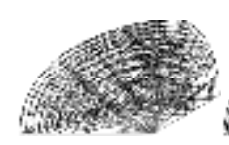

$n=3$

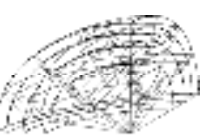

$n=2$

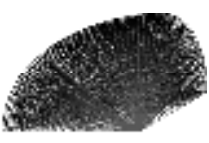

$\mathrm{n}=4$
Fig.1 The 3D visualization of octant grid subdivision based on octree(Left) and the 3D visualization of octant grid subdivision based on unqueal octree(right)

The mesh scheme has the following characteristics: 1) a simple grid cell shape. There are two kinds of grid cell shape: near the center of the sphere are spherical triangular pyramids, and the remaining layers are all spherical triangular prismoids. All grid cells (upper and lower) is a spherical bottom, sides are flat. Similar to the shape of the grid in the same layer, the nature and magnitude of the volume deformation is equivalent to the area deformation of corresponding spherical grid (that is, the bottom surface of the grid elements). Different with the volume of the same column grid. 2) Arranged in neat grid system, the spatial relationship between the units is simple and consistent. All layers of grid cells neat, uniform, compact, symmetrically arranged around the center of the sphere. Horizontal grid cells in the same layer, their geometric center are evenly distributed in the same sphere, and the shape is similar, so they keep the circle structure of the Earth; radial grids geometric center of the same column and their bottom are all located on a diameter of the sphere and correspond to neat. On the whole, the spatial relation(topological) of each layer grid cell are almost identical (completely octree), so it is of great significance for the grid cell coding and spatial relation reasoning. 3) Spatial grid distribution and geocentric Cartesian coordinate system corresponds neatly, but no significant relationship with latitude and longitude grid. However, it can realize the mutual transformation between the grid coding and conventional the coordinate system by a specific coding and decoding method. Through knowledge of spherical geometry, the above octree grid cell volume formula can be deduced:

$V=(A+B+C-\pi)\left(r_{1}^{3}-r_{0}^{3}\right) / 3=(\theta-\pi)\left(r_{1}^{3}-r_{0}^{3}\right) / 3$

Wherein: $A, B, C$ are three angles of spherical triangle; $r_{l}, r_{0}$ is the radius of the inner and outer sphere of the cell bottom (if it is the triangular pyramids, $r_{0}$ is equal to 0$), \theta$ is the sum of the triangle angles. According to this formula, we can easily derive the cells volume ratio formula of same layer and column (Jinxin, W., et al, 2013). According to the formula, octree equidistant grid cells in the same layer, their convergence value of maximum and minimum volume ratio is 2 (Jinxin, W., et al, 2013). When the recursive subdivision have done 8-9 times, the unit volume of deformation will be basically stable; radially, the volume ratio of outermost triangular prismoid and the corresponding triangular pyramid in center is divergent. According to the same formula for the volume ratio of the column grid cell, set its value equal to 1 , the radial ball radius split sequence that each cell volume is equal can be obtained. It is: $\sqrt[3]{1 / 2^{n}}, \sqrt[3]{2 / 2^{n}}, \sqrt[3]{3 / 2^{n}} \ldots \sqrt[3]{2^{n}-1 / 2^{n}}$. If the radius of the sphere is subdivided according to that percentage, rather than equal portions, then you can get variable length octree subdivision(Right of Fig.1). This unequal-length octree subdivision system in addition to has the characteristics of equal length octree system, but also has a merit of radial grid cells size equal (Jinxin, W., et al, 2013). For a given level of subdivision, the grid system of the ratio of maximum and minimum convergence value is 2 , and the corresponding values of degradated octree is 8.89 (Lixin, W, et al, 2009), shown in Figure 2 . 


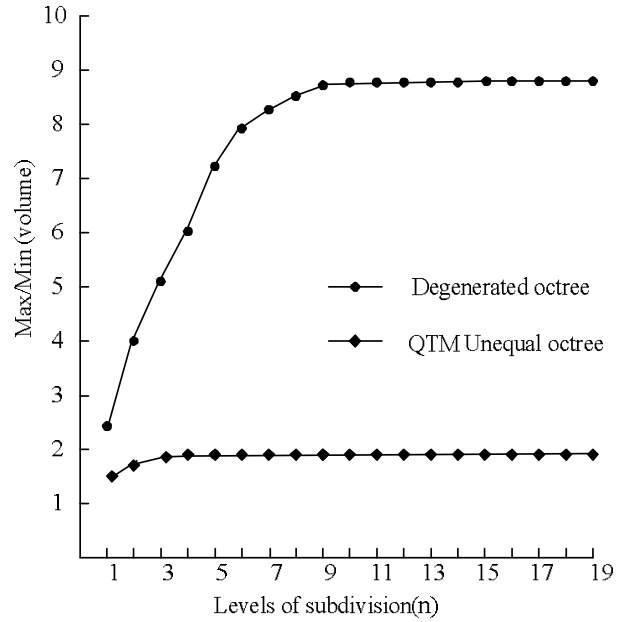

Fig. 2 Comparison of distortion ratio of grid's volmne between Unequal octree and degenerated octree

In addition, from the view of differences of area and side length of spherical cells at the same layer, octree system are better than degradated octree system; while the radial edge length difference on the contrary, unequal length octree vary greatly, which is as a consideration in exchange for the greatest degree of overall grid size is relatively homogeneous (Jinxin, W., et al, 2013). Degradated octree system stick the traditional latitude and longitude grid, a more direct correspondence with latitude and longitude, and the octree system neatly corresponds to 3D Cartesian coordinates, and thus, these two programs each has its advantages.

\subsection{Encoding and decoding method}

Grids coding and its relationship with conventional Cartesian coordinate system conversion is a key issues on discrete global grids. Lots of research results have been achieved in this field. On QTM concerned, there are many coding methods, such as Fixed Direction coding(Goodchild, M F., et al, 1992), ZOT coding(Dutton, 1989), LS coding(Lee, M., et al, 2000), SQC coding(Otoo,et al, 1993), etc. For 3D degradation octree grids, Jieqing Y., et al(2009) proposed SDZ and MDZ coding. By the analysis of the mesh encoding and decoding principle, we found that spherical quadtree plus radial binary tree coding is a convenient and effective way to the $3 \mathrm{D}$ octree grids.

This coding method consists of two parts: the spherical twodimensional code and the radial depth code. Spherical twodimensional code can be any one of above Fixed Direction, ZOT, LS and SQC codes, and they all belong to the quadtree code. It is used to determine the lateral position of the grid cells. In aliquots, for a example, the radial binary encoding mechanism shown in Figure 3. It is very simple to determine the depth position of the grid cells.

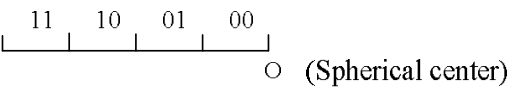

Fig. 3 The encoding method of radial binary tree $(n=2)$

The biggest advantage of this approach is to fully utilize the existing research results and the geometric properties of ball and cube are homeomorphic. Taking Goodchild's Fixed Direction code as a example, a code with latitude and longitude and depth mutual conversion is achieved in the paper

(Fig.4).

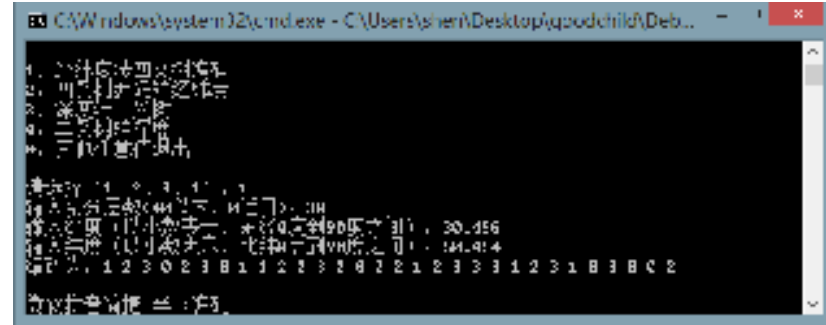

Fig.4 Coding Conversion Example

\section{APPLICATIONS OF GREAT CIRCLE ARC QTM OCTREE MODEL}

\subsection{Surface entity modeling}

Here we talk about how to use the 3D octree grid to achieve global or large regional DEM visualization. After obtaining the traditional DEM data based on latitude and longitude grid: 1) According to DEM grid scale, setting a particular split-level, find the latitude and longitude coordinates of each grid point; 2 ) Interpolating all grid points elevation; 3) Transforming the geographical coordinates of the grid points into threedimensional Cartesian coordinates; 4) Plotting grid points, and sequentially connected (Fig. 5).

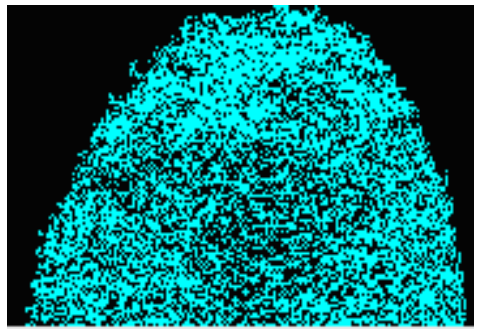

Fig. 5 Large area DEM visualization 4.2 Underground and aerial entity modeling

Underground and aerial entity modeling, must be based on true three-dimensional spatial data model; For ESSG grid system is concerned, it must realize the true three-dimensional body on the earth split, which is based on the grid "voxel" to achieve three-dimensional construction of geographical entities mode. Since octree subdivision scheme has clear mathematical relationship, you can easily build the relationship between each grid code and its corresponding voxel (vertex coordinates), therefore, it can be achieved voxel-based solid modeling ground or air.1) According to the size of geographic entities and the accuracy of expression, to determine the size of the voxel, and then determine the level of recursive subdivision; 2) According to the coordinates of the feature points outside entity, to compute the corresponding voxel grid codes, and then to determine the position and geometry of each corresponding grid; 3) Plotting all the physical area corresponding grid, to achieve a three-dimensional solid model visualization. Fig. 6 is a schematic view of an underground ore body model, and Fig.7 (next page)is a schematic model of the satellite orbit. 


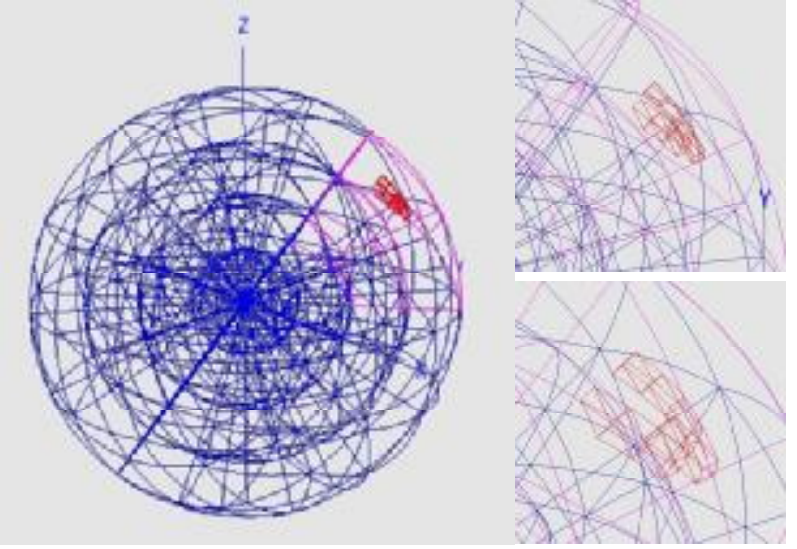

Fig.6 An underground ore body model

It should be noted that we here are examples of the scheme's initial application. Further in-depth applications will be the directions of our future research.

\section{CONCLUSIONS}

DGGs and ESSG are all the spatial data models for large-scale regional and global at the background of Digital / Smarter Earth, especially the ESSG achieves a three-dimensional global gravity field split, and it can integrate whole geospatial data.Our studies have shown that 3D global QTM octree grid model has the potential ability of inegration and management of surface, underground and aerial multi-resolution spatial data, is a effective spatial data model oriented global GIS and Digital Earth, and has broad application prospects.

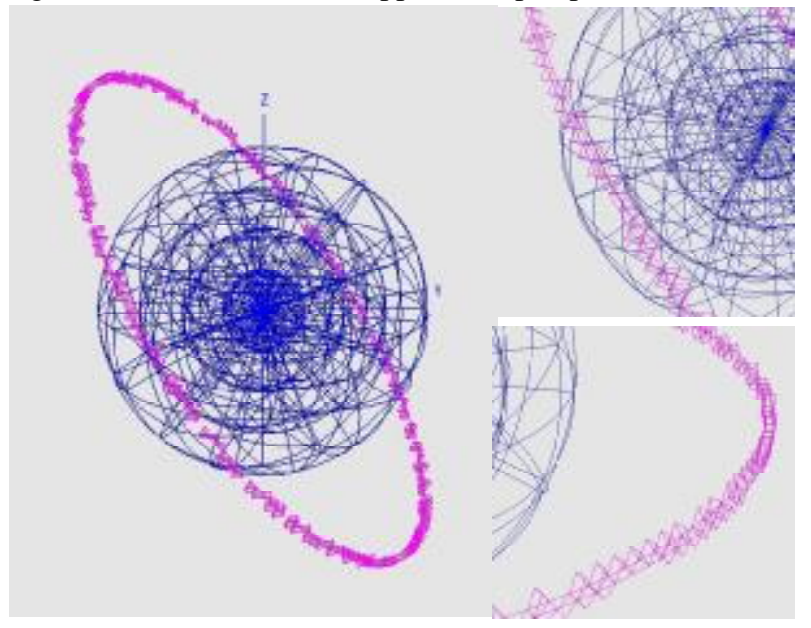

Fig. 7 A model of the satellite orbit.

The following three aspects are our future research directions: 1) How to achieve the massive, multi-scale, multi-temporal and multi-form geospatial data for effective integration, integration and management based on 3D global QTM octree grid; 2) Related queries, retrieval and application algorithms design and implementation; 3) How to build a platform facing the complex geography computing of large-scale and global issues combined the $3 \mathrm{D}$ global discrete grids to grid computing, cloud computing ,etc..

Acknowledgments. This research is funded by State Key Laboratory for Information Engineering in Surveying, Mapping and Remote Sensing, China (12I02), Key Laboratory of Mine Spatial Information Technologies State Bureau of Surveying, Mapping and Geographic Information, China(KLM201203), and Students Innovative Experimental Projects, Chinese Ministry of Education(201310459046).

\section{Reference}

[1] Ballard, S., Hipp, J R., Young, C J., 2009. Efficient and accurate calculation of ray theory seismic travel time through variable resolution 3D Earth models. Seismological Research Letters, 80(6), pp. 989-999.

[2] Baumgardner, J R., 1985. Three-dimensional treatment of convective flow in the earths mantle. Statistical Physics, 39(5),pp. 501-511.

[3] Dutton, G., 1989. Modeling location uncertainly via hierardhical tessellation, In: Accuracy of spatial database. Tayor and Francis, London, Britain, pp. 125-140.

[4] Goodchild, M F., 1994. Criteria for evalution of global grid models for environmental monitoring and analysis. NCGIA Technical Report 94. http://www.ncgia.ucsb.edu/pubs/pubslist.html (12， May, 2000).

[5] Goodchild, M F.2000. DISCRETE GLOBAL GRIDS FOR DIGITAL EARTH. International Conference on Discrete Global Grids, 2000 - ncgia.ucsb.edu.

[6] Goodchild, M F, Yang, S., 1992. A hierarchical data structure for global geographic information systems. Computer Vision and Geographic Image Processing, 54(1), pp. 31-34.

[7] Jieqing, Y., Lixin, W., 2009. On Coding and Decoding for Sphere I)egenerated-Oetree Grid [J]. Geography and GeoIrdormation Science, 25(1), pp. 5-9, 31.

[8] Jinxin, W., Fengnian, L., Jie C., 2012. Comparison of surface and solid discrete global grids. Science of Surveying and Mapping, 37(6), pp. 34-36.

[9] Jinxin, W., Fengnian, L., Tongde, G., Jie, C., 2013. Global 3D-Grids Based on Great Circle Arc QTM Sphere Octree and Unequal octree. Geomatics and Information Science of Wuhan University, 38(3), pp. 344-348.

[10] Kageyama, A., Sato, T., 2004. The "Yin-Yamg Grid": An overset grid in spherical geometry. Geochemistry Geophysics Geosystem, 5(9), pp. 1-15.

[11] Kimerling, A J., Sahr, K., White, D., 1999. Comparing geometrical properties of global grids. Cartography and Geographic information Science, 26(4), pp. 271-288.

[12] Lee, M., Samert, H., 2000. Navigating through Triangle meshes implemented as Linear Quadtree. ACM transactions on Graphics, 19(2), pp. 79-121.

[13] Lixin, W, Jieqing, Y., 2009. GlobaI 3DGrid Based on Sphere Degenerated Octree and Its Distortion Features. Geography and Geo-Irdormation Science, 25(1),pp.1-4.

[14] Lixin, W., Jieqing, Y., 2012. Earth System Spatial Grid and Its Application Models. Geography and GeoIrdormation Science, 28(1),pp. 7-13.

[15] Otoo, E., Zhu, H., 1993. Indexing on spherical surfaces using semi-quadcodes. In: Advances in spatial databases $3^{\text {rd }}$ International Sympusium, Springer- Verlag, Berlin, pp. 509-529.

[16] Stadler, G., Gurnis, C., Burstedde, C., 2010, The dynamics of plate tectonics and mantle flow: From local to global scales. Science, 329(5995),pp. 1033-1038.

[17] Tsuboi, S., Komatitsch, D., Ji, C., 2008. Computations of global seismic wave propagation in three dimensional Earth mode. In: High-performance,pp. 434-443.

[18] Xuesheng, Z., Miaole, H., Jianjun, B., Spatial Digital Modeling of the Global Discrete Grids. Press of Surveying and Mapping, Beijing, pp. 68-70. 
International Archives of the Photogrammetry, Remote Sensing and Spatial Information Sciences, Volume XL-4/W2, 2013 ISPRS WebMGS 2013 \& DMGIS 2013, 11 - 12 November 2013, Xuzhou, Jiangsu, China

Topics: Global Spatial Grid \& Cloud-based Services

[19] Yongsheng, Z., Jin B., Xiaochong, T., 2007. Geospatial Information spherical discrete grid - theory, algorithms and applications. Science Press, Beijing, pp. 5-9.

[20] Zhao, X. S., Chen, J., \& Li, Z. L., 2002. A QTM-based algorithm for the generation of Voronoi diagram on sphere. Advances in spatial data handling. Springer-Verlag, Berlin, pp.269-284. 